\title{
Intelligent Microscopy: A Path Toward Tailored Materials at the Atomic Scale
}

Mitra Taheri, James Hart and Cassandra Pate

Johns Hopkins University, United States

In situ transmission electron microscopy (TEM) and electron energy-loss spectroscopy (EELS) are powerful tools for the observation of real-time materials processes. Specifically, in situ TEM is a reliable source for probing dynamic phenomena in order to gain a predictive understanding of myriad materials and use this knowledge to tailor future, improved systems. The development of radiation hard direct detection (DD) electron sensors has enabled improvements in the quality of in situ data for TEM imaging, and recently, we have demonstrated that DD provides far-reaching benefits for EELS [1]. Specifically, the sharper point spread function and reduced pixel size of DD provides a significant improvement in combined energy resolution and field of view (FOV). With these improvements, critical issues in both physical and biological sciences can be addressed. Additionally, dynamic ionic or defect-dependent phenomena can be quantified with the combination of in situ methods and the DD EELS system [1-3].

This talk reviews recent work in using in situ investigation of chemistry-property relationships and coordination chemistry in a rapidly emerging family of 2D materials, MXenes. MXenes are a family of 2D materials with potential applications such as energy storage, electromagnetic interference shielding, catalysis, and wireless electronics. Their general formula is $\mathrm{M}_{n+1} \mathrm{X}_{n} \mathrm{~T}_{x}$, where $\mathrm{M}$ is a transition metal, $\mathrm{X}$ is $\mathrm{C}$ and/or $\mathrm{N}, n=1-4$, and $\mathrm{T}$ represents the surface termination (-OH, $-\mathrm{F}$, and $-\mathrm{O})$. The ability to tune or modify MXene surface termination is a goal for controlling functional properties of these materials, such as metallic conductivity, work function, charge storage properties, and superconductivity [2,3]. Here we highlight the thermal control of $-\mathrm{F}$ termination in $\mathrm{Cr}_{2} \mathrm{TiC}_{2} \mathrm{~T}_{x}$ and offer a first step toward termination engineering this MXene for magnetic applications, which demonstrates high energy electron spectroscopy as a powerful approach for surface characterization in 2D materials.

Finally, an outlook on emerging time resolved studies and key challenges for "big data" will be presented [4]. In particular, a major challenge in understanding complex phase transformations is obtaining high resolution spectra at rapid time scales. Even with direct detection, spectra can be highly noisy. To combat this, use of machine learning for rapid acquisition EELS will be presented in the context of accuracy of identification of oxidation state and coordination chemistry. This work will be discussed as a foundation for future integration of ML at high speeds during in situ TEM experiments. 


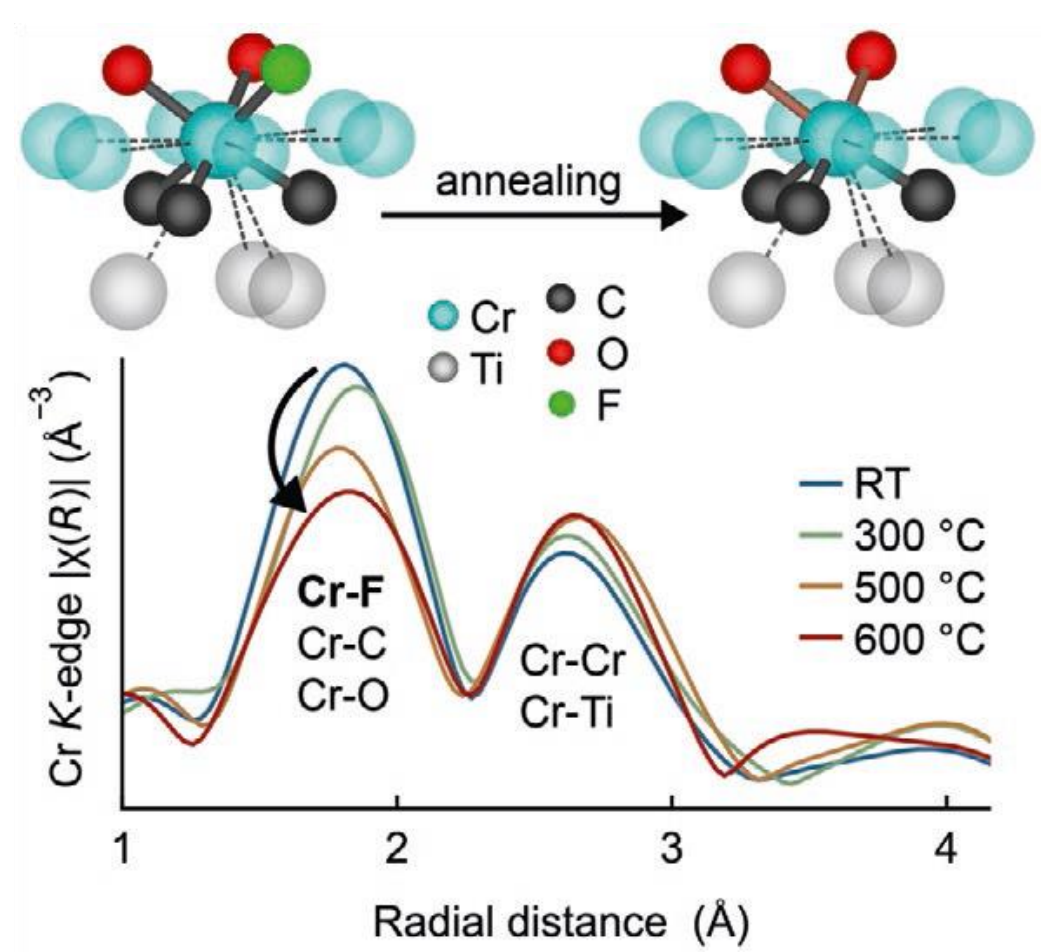

Figure 1. Figure 1. Coordination chemistry as a function of temperature, from [3].

\section{References}

[1] Hart, J.L., Lang, A.C., Leff, A.C., Longo, P., Trevor, C., Twesten, R.D. and Taheri, M.L., 2017. Direct detection electron energy-loss spectroscopy: a method to push the limits of resolution and sensitivity. Scientific reports, 7(1), pp.1-14.

[2] Hart, J.L., Hantanasirisakul, K., Lang, A.C., Anasori, B., Pinto, D., Pivak, Y., van Omme, J.T., May, S.J., Gogotsi, Y. and Taheri, M.L., 2019. Control of MXenes' electronic properties through termination and intercalation. Nature communications, 10(1), pp.1-10.

[3] Hart, J.L., Hantanasirisakul, K., Lang, A.C., Li, Y., Mehmood, F., Pachter, R., Frenkel, A.I., Gogotsi, Y. and Taheri, M.L., 2021. Multimodal Spectroscopic Study of Surface Termination Evolution in Cr2TiC2Tx MXene. Advanced Materials Interfaces, p.2001789.

[4] Spurgeon, S.R., Ophus, C., Jones, L., Petford-Long, A., Kalinin, S.V., Olszta, M.J., Dunin-Borkowski, R.E., Salmon, N., Hattar, K., Yang, W.C.D., Sharma, R...... and Taheri, M.L., 2020. Towards data-driven next-generation transmission electron microscopy. Nature materials, pp.1-6. 